\title{
Anatomical Analysis of the Developing Cerebral Vasculature in Premature Neonates: Absence of Precapillary Arteriole-to-Venous Shunts
}

\author{
JOHN A. ANSTROM, WILLIAM R. BROWN, DIXON M. MOODY, CLARA R. THORE, \\ VENKATA R. CHALLA, AND STEVEN M. BLOCK \\ Department of Radiology [J.A.A., W.R.B., D.M.M., C.R.T.], Department of Pathology [V.R.C.], \\ Department of Pediatrics [S.M.B.], Program in Neuroscience [J.A.A., W.R.B., D.M.M.], Wake Forest \\ University School of Medicine, Winston-Salem, North Carolina, U.S.A.
}

\begin{abstract}
Germinal matrix hemorrhage-intraventricular hemorrhage (GMH-IVH) commonly affects premature neonates. The gravity of the consequences associated with GMH-IVH makes it a major concern in their care. GMH-IVH begins in the germinal matrix tissue and is, most commonly, the result of venous rupture. Arteriole-to-venous precapillary shunts in the cerebrum of premature neonates could, if present, lead to elevated venous pressure in the germinal matrix and, thus, would be an important etiological factor. We report an autopsy study, involving 33 cases of premature neonates, designed to determine whether precapillary arteriole-to-venous shunts are present in the cerebral vasculature. Brain tissue was embedded in celloidin, sectioned into $100-\mu \mathrm{m}$-thick slices and stained using alkaline phosphatase enzyme histochemistry, a method that distinguishes afferent from efferent vessels. Our sections, which are ideal for tracing vessels
\end{abstract}

over long distances and for displaying patterns of branching and connections with other vessels, indicate that precapillary arteriole-to-venous shunts are not a major influence on cerebral blood flow in babies born at 23 wk gestation or later. The cerebral vasculature in one baby, who died at $24 \mathrm{wk}$ postconception, included shunt-like connections, whereas in 34 babies shunts were not identified. We conclude that precapillary arteriole-to-venous shunts are not a significant factor leading to GMH. (Pediatr Res 52: 554-560, 2002)
C IV Abbeviations
GMH-IVH, germinal matrix hemorrhage-intraventricular hemorrhage
AP, alkaline phosphatase

GMH-IVH is the most common intracranial hemorrhage afflicting premature neonates (1). The incidence, though declining, is about $1.45 \%$ of live births or about 58,000 cases per year. However, the incidence is highest in the smallest premature babies and, with survival rates increasing for this group, GMH-IVH remains, and will remain, a major focus of concern in neonatal intensive care units. In addition to the high number of cases, the gravity of the consequences associated with hemorrhagic damage make GMH-IVH a major concern in the care of premature neonates. Understanding the etiology and neuropathology of GMH-IVH is a necessary prerequisite for maximizing the benefits of current care and for discovering preventive measures $(1,2)$.

It is widely accepted that GMH-IVH begins in the germinal matrix tissue (1). However, descriptions of GMH-IVH neuro-

Received October 31, 2001; accepted March 4, 2002.

Correspondence: John A. Anstrom, Ph.D., Department of Radiology, Wake Forest University School of Medicine, Winston-Salem, NC 27157, U.S.A.; e-mail: janstrom@wfubmc.edu.

Supported by NIH grants NS20618 and NS36780 to D.M.M.

DOI: 10.1203/01.PDR.0000030877.20888.F6 pathology vary considerably, especially in reference to the identity of the ruptured vascular element. The vascular origin of a hemorrhage is difficult to identify in histologic preparations because of the unusual structural qualities and high concentration of vessels within the germinal matrix tissue. Thus, even when a ruptured vessel is identified, designating it as an arteriole, capillary, or vein can be problematic and, in fact, all three vascular components have been implicated as sites of hemorrhagic origin (1,3-10).

Ghazi-Birry et al. (10) studied a series of neonatal brains that contained hemorrhagic foci. In this study, they used a specialized histochemical technique that distinguishes veins from arteries and capillaries and demonstrated that the overwhelming majority of hemorrhages were of venous origin. These results imply that GMH-associated etiologic factors that elevate venous pressure are of paramount importance and must be monitored and controlled if hemorrhage is to be minimized.

Elevated venous pressure leading to venous rupture could be the result of either impaired venous outflow from the germinal matrix or elevated arterial pressure. Both of these conditions 
have been identified as etiological factors leading to GMHIVH (1).

Intravascular pressure, under normal circumstances, falls markedly when blood leaves arterioles and enters capillaries. However, if arterioles are connected directly to veins by way of precapillary channels, or shunts, this drop in pressure would not occur, and arterial pressure would pass, more or less unabated, directly to the venous circulation. Medullary veins in the white matter accommodate flow either centrally or peripherally. Therefore, elevated pressure in these vessels could pass centrally to the veins of the germinal matrix.

The adult cerebral vasculature has been studied extensively in an effort to identify shunts. Bell et al. (11) and Moody et al. (12) concluded that such connections were not present. Interpretation of results from anatomical studies of the fetal cerebral vasculature have differed in regard to the presence and significance of shunts $(13,14)$. Nelson et al. (14) described anastomotic vessels up to $40 \mu \mathrm{m}$ in diameter connecting transcerebral channels in the cerebrum of 20 -wk gestation fetuses. It is not known whether these putative shunts persist into developmental stages where they could influence postnatal circulation. Several laboratories have obtained evidence of fetal shunts but have minimized the significance of this finding (15-17). In contrast, Moody et al. (18) could find no evidence for the existence of arteriolar-to-venous shunts in the cerebral vessels of a 31-wk gestation neonate. Because fetuses as young as 23 wk gestation routinely survive (but exhibit a high incidence of $\mathrm{GMH}$ ), it is of interest to determine whether anastomotic shunts between arterioles and veins exist at these and later developmental stages. The existence of shunts at these stages could have a major influence on the progression to GMH-IVH.

This present study is a systematic effort to identify shunts connecting arterioles with veins in the cerebral hemispheres of premature neonates. We studied an autopsy series of brains from neonates born between 23 and 36 wk gestation. Whole brain slices were embedded in celloidin and cut into sections $100-\mu \mathrm{m}$ thick. These sections are more than 10 times thicker than the paraffin sections used for routine neuropathological examination. Individual vessels in these uncommonly thick slices can be traced over extended distances and complex connections between vessels can be identified. In addition, the sections were stained using AP enzyme histochemistry, which distinguishes afferent from efferent vessels (11). These preparations, which enable one to follow vessels over long distances, to view connections between neighboring vessels, and to classify connecting vessels as either afferents or efferents, are ideally suited for the identification of arteriovenous shunts.

\section{MATERIALS AND METHODS}

Brains from 35 premature neonates obtained at autopsy were studied. Postconception ages, taken from maternal medical records, ranged from $22-23$ wk to $43 \mathrm{wk}$ and postnatal survival times ranged from hours to over 3 mo. This information and additional clinical data are summarized in Table 1. Throughout this report, subjects are categorized according to their postconception age, which we define as the sum of the gestational age and survival time as obtained from the medical history.
Preparation of tissue. As soon as possible after autopsy, the neonatal brains were fixed in cold $70 \%$ alcohol for $10-12 \mathrm{~d}$. Subsequently, a whole-brain slice approximately $1-\mathrm{cm}$ thick, incorporating both hemispheres joined by the corpus callosum, was obtained from each brain at and posterior to the foramen of Monro and included the thickest mass of germinal matrix as well as basal ganglia, thalamus, centrum semiovale, and cortex. The brain slices were then dehydrated in ascending grades of ethanol, embedded in celloidin, and serially sectioned at 100 $\mu \mathrm{m}$ on a base sledge microtome. The sections were stained for native endothelial AP by Bell and Scarrow's modification of the Gomori method $(11,19)$. In addition, a subset of these slides were also stained by standard histologic methods including hematoxylin and eosin, cresyl violet acetate/light green, and trichrome.

Method of tabulation. Our observations covered both cerebral hemispheres of each specimen and included cortex, subcortical white matter, and periventricular white matter, as well as the basal ganglia and thalamus. The search began at the optic tract and progressed counterclockwise around the surface of the specimen until 25 arterioles and their connections had been examined. Two to four sections were generally required to accumulate these 25 examples. All vessels in the sections were examined. Many were cut at an angle to their long axis, which precluded detection of their connections. However, when an arteriole appeared in longitudinal profile, all branches would be followed as far as possible, either to their cut end or to their juncture with an adjacent arteriole or vein. We would then tabulate the connections and note whether or not the connecting channels included capillaries. If capillaries were included, the connection was regarded as a demonstration of typical vascular connections, i.e. arteriole to capillary. If the connecting channel did not include capillary-sized vessels, the connection would be considered a shunt. At least 25 examples were tallied sequentially from each case.

Measurements of the smallest appearing vessels, which were considered capillaries, indicated that in our specimens, these channels measured approximately $10 \mu \mathrm{m}$ in diameter and had a range of $6-11 \mu \mathrm{m}$. Arterioles were characterized as having a diameter larger than $10 \mu \mathrm{m}$, being $\mathrm{AP}^{+}$, and, in most instances, oriented perpendicular to the brain surface. Veins were also larger than $10 \mu \mathrm{m}$ and were $\mathrm{AP}^{-}$. Because we used crosssections of the entire brain (or one cerebral hemisphere in the case of older specimens), the anatomical relationship of vessels to the brain surface and lateral ventricles was readily evident and could assist in determining their identity.

\section{RESULTS}

Neonates $>30 w \boldsymbol{k}$ postconception. Brain slices obtained from babies $30 \mathrm{wk}$ postconception or later and stained with the AP histochemical method revealed a vascular pattern similar to that seen in adult brain specimens, where arterioles and capillaries are stained intensely, but veins are unstained or lightly stained. Penetrating arterioles were observed extending from the brain surface, near their presumed origin from leptomeningeal arteries, toward the lateral ventricle (Fig. 1). The origin of these vessels at the brain surface, their diameter, which 
Table 1. Case presentation

\begin{tabular}{|c|c|c|c|c|}
\hline Case no. & Postconception age (wk) & Survival (d) & GMH & Medical complications \\
\hline 1 & $22-23$ & 2.5 & + & RDS \\
\hline 2 & 23 & 3 & - & RDS, PDA \\
\hline 3 & 24 & 0 & + & RDS, PIE, pneumothorax \\
\hline 5 & 24 & $<0.5$ & + & RDS, PIE, HMD \\
\hline 6 & 24 & $<0.5$ & + & RDS, HMD, tension pneumothorax \\
\hline 7 & 24 & $<0.5$ & - & RDS, PIE \\
\hline 10 & 25 & 1.5 & + & RDS, tension pneumothorax, PIE \\
\hline 11 & 25 & 2 & + & RDS, PIE, pulmonary hemorrhage \\
\hline 12 & 25.5 & 7 & - & RDS, NEC \\
\hline 13 & 26 & 0 & + & RDS, HMD \\
\hline 14 & 26 & 14 & - & NEC, RDS, bilateral pneumothoraces \\
\hline 15 & 27.5 & 14 & + & Bilateral pneumothoraces, bronchopneumonia \\
\hline 20 & 30 & 15 & & Sepsis \\
\hline 21 & 30 & 30 & & RDS, PDA, pulmonary edema, NEC \\
\hline 22 & 30 & 39 & & RDS, PIE, PDA, pnuemothorax, NEC, sepsis \\
\hline 23 & 31 & 22 & & Pulmonary hemorrhage and hypertension \\
\hline 24 & 31.5 & 1 & & RDS, bilateral pneumothoraces, HMD \\
\hline 25 & 33 & 42 & - & RDS \\
\hline 26 & 33 & 61 & + & RDS, BPD, PDA \\
\hline 27 & 34 & 49 & - & NEC, probable pseudomonas sepsis \\
\hline 28 & 35 & 6 & + & RDS, PDA, lung consolidation \\
\hline 29 & 36 & 1 & & Hypoplastic lungs, pneumothorax \\
\hline 30 & 39 & 4 & & Left ventricular hypoplasia, PDA, PIE \\
\hline 31 & 41 & 4 & & Perinatal asphyxia (hypoxic/ischemic injury) \\
\hline 32 & 42 & 18 & & Down's syndrome, pulmonary hypertension, RDS, PDA, ECMO \\
\hline
\end{tabular}

RDS, respiratory distress syndrome; PDA, patent ductus arteriosus; PIE, pulmonary/interstitial/emphysema; HMD, hyaline membrane disease; NEC, necrotizing enterocolitis; BPD, bronchopulmonary dyspasia; ECMO, extra corporeal-membrane oxygenation.

exceeds $10 \mu \mathrm{m}$, and their staining with AP confirmed their identity as afferents. A structurally complex network of afferent vessels was revealed in the cortex, where an extensive capillary network was observed between the array of penetrating arterioles. Adjacent arterioles supplied a shared intervening capillary bed (Fig. 1). It was possible to trace routes that led from one arteriole, into the capillary bed, and on to a neighboring arteriole. But, in these instances, there also existed vascular pathways connecting the capillary bed to veins. Neighboring arterioles connected by vessels larger than capillaries were not observed. We concluded, therefore, that shunt-like connections were not present. Similarly, connections between arterioles and nearby veins always included capillaries.

Cerebral subcortical white matter was characterized by arterioles that extended from the overlying cortex toward the lateral ventricle (Fig. 2A). The density of capillaries between adjacent arterioles was less in the white matter than in the cortex. Nevertheless, vascular channels connecting one arteriole to another by way of intervening capillaries were readily identified in the white matter. Most commonly, the route through the capillary bed was complex, involving numerous branch points though, occasionally, a capillary lacking branches joined neighboring arterioles. Arterioles and veins were also observed connected by similar capillary-sized connections. These connections, too, were most often complex in that they had multiple branch points along their length, though simple capillary routes without branch points were also observed. In the 16 specimens of this age group, shunts connecting arterioles with veins were not observed.

The branching pattern displayed by arterioles in the deep white matter of neonates $>30$ wk postconception was considerably more complex than the pattern seen in our younger specimens. Figure $2 B$ demonstrates an arteriole in the periventricular white matter of a brain from a $36 \mathrm{wk}$ postconception baby. This arteriole displays at least five orders of branching. This complexity, which results in a dramatic increase in capillary density, would markedly decrease the likelihood of maintaining short, direct, shunt-like connections between white matter arterioles and their neighboring veins.

Neonates at 27-30 wk postconception. Neonates 27-30 wk postconception had a cortical vascular network that resembled that of term neonates where the parallel profiles of penetrating arterioles were separated by an extensive capillary network (Fig. 3A). Neighboring arterioles supplied a common capillary bed. Connections between neighboring arterioles could be identified readily and these pathways were found always to 

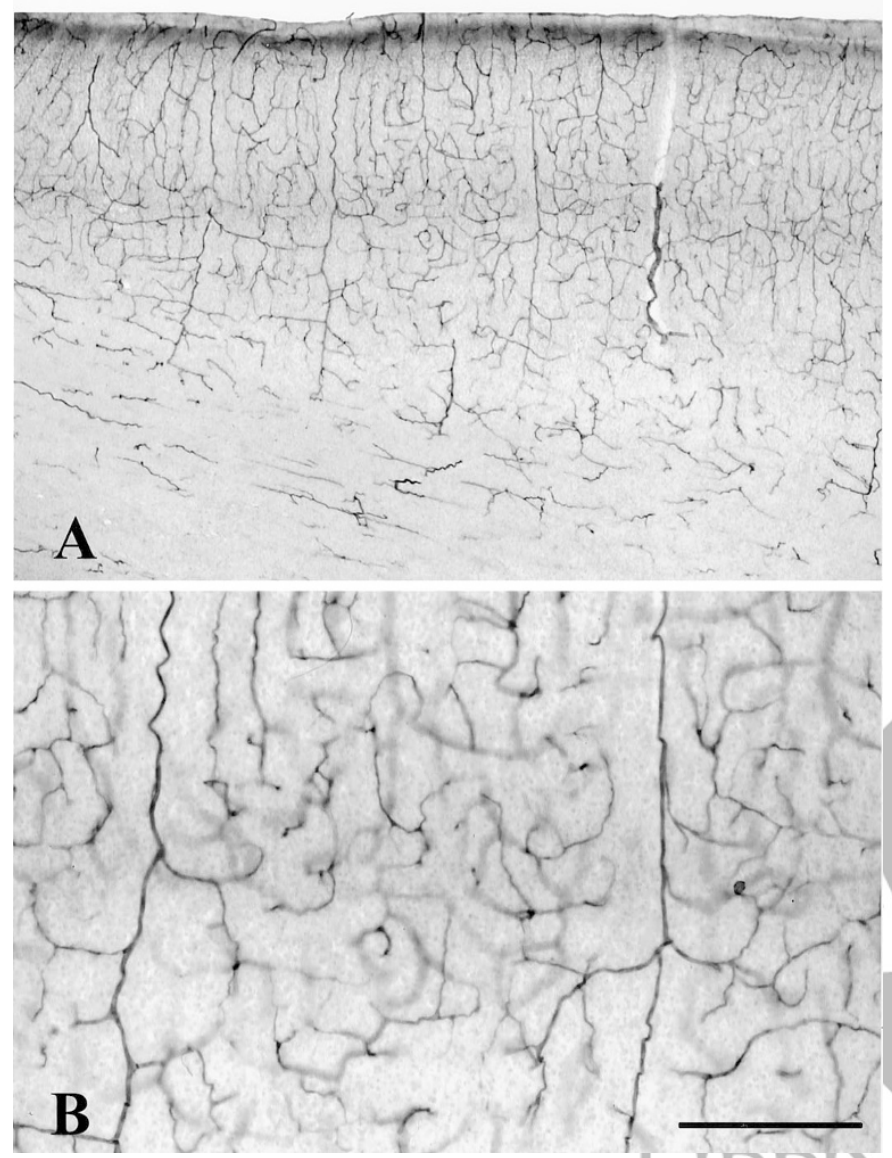

Figure 1. AP enzyme histochemistry of $100-\mu$ m-thick celloidin section from brain of a $36 \mathrm{wk}$ postconception neonate that survived $1 \mathrm{~d}$. (A) $\mathrm{AP}^{+}$arterioles arise from the surface of the cerebral hemisphere and penetrate into the parenchyma. Many of the arterioles extend through the cortex to enter the white matter. Within the cortex, many branches connect to the intervening capillary network. Adjacent arterioles are connected through a well-developed capillary bed. $(B)$ Adjacent arterioles are connected to a shared capillary bed. Bar $=625 \mu \mathrm{m}$ in $A ; 250 \mu \mathrm{m}$ in $B$.

include capillary-sized channels. Occasionally, short, capillary-sized channels lacking branches joined two adjacent cortical arterioles, but this arrangement was not common.

The white matter vascular pattern in the 27-30 week age group also resembled the pattern seen in older specimens. Longitudinal profiles of arterioles descending into the white matter from the overlying cortex were observed extending toward the lateral ventricle. Adjacent arterioles were generally connected through a complex network of vessels that included capillaries (Fig. 3B). Arterioles connected to veins by channels that lacked branches were common, but these connecting channels were the size of capillaries and would not, therefore, be considered shunts. In this age group, shunt-like profiles where arterial blood could pass directly into a vein without first coursing through a capillary were not observed.

Neonates $<27$ weeks postconception. We have collected 35 autopsy specimens for our analysis of cerebrovascular development in the human premature neonate. During the course of our work, it was discovered that endothelial cells of arterioles and capillaries within the cerebral white matter do not express a full complement of AP until approximately the 28th gesta-

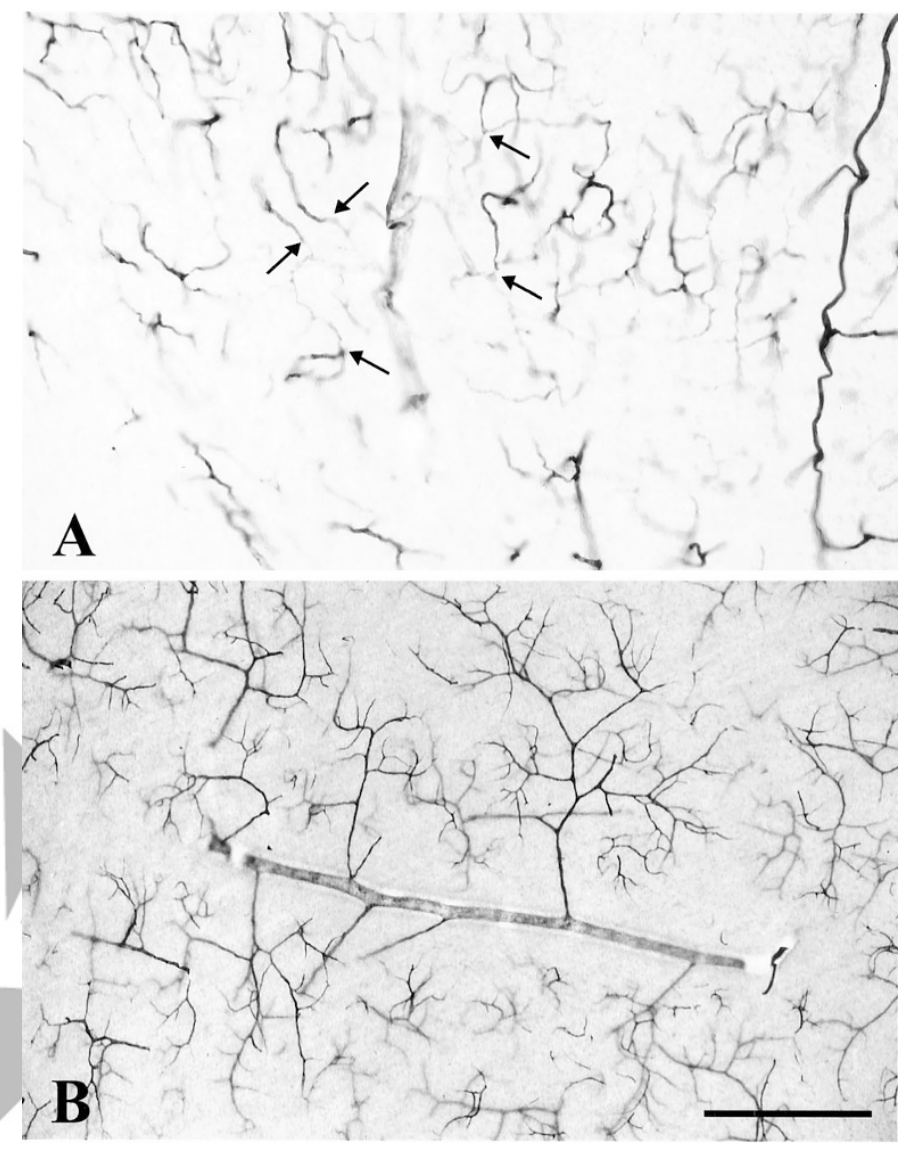

Figure 2. AP enzyme histochemistry of $100-\mu$ m-thick celloidin section from brain of a 36 wk postconception neonate that survived $1 \mathrm{~d}$. (A) At the boundary between cortex and white matter, postcapillary venules connect to a vein. The junctions of stained capillaries and postcapillary venules are identified (arrows). The unstained postcapillary venules connect to a large vein. Veins are either unstained or as in this case, lightly stained. $(B)$ AP histochemistry counterstained with cresyl violet acetate/light green. In the deep white matter a large arteriole branches into several orders of subdivisions. Arterioles feed a complex capillary network. Section is counterstained with cresyl violet acetate/ light green. Bar $=250 \mu \mathrm{m}$.

tional week (20). Nevertheless, vascular connections can be analyzed in the cortex and in those white matter vessels that do stain. The cortical afferents formed a network with considerably less branching than is observed in older specimens (Fig. 4). In these younger specimens, adjacent cortical arterioles often were joined by either straight, capillary-sized channels with no branches leading to other capillary beds, or by capillary-sized channels that branched into a comparatively simple capillary bed. A minority of the $\mathrm{AP}^{+}$cortical arterioles in these young babies extended into the subcortical white matter.

$\mathrm{AP}^{+}$arterioles in the white matter were most concentrated in this subcortical region and were sparse in the deep, periventricular white matter. Where it was possible to trace vascular channels between adjacent arterioles or between an arteriole and a vein, the pathways inevitably included a capillary. However, in one specimen, several examples of shunts joining arterioles to veins were identified. Figure 5 illustrates this particular specimen, which is from case 8. Two different shunt-like connections between subcortical arterioles and an adjacent $\mathrm{AP}^{-}$vein can be observed. The connecting channels 


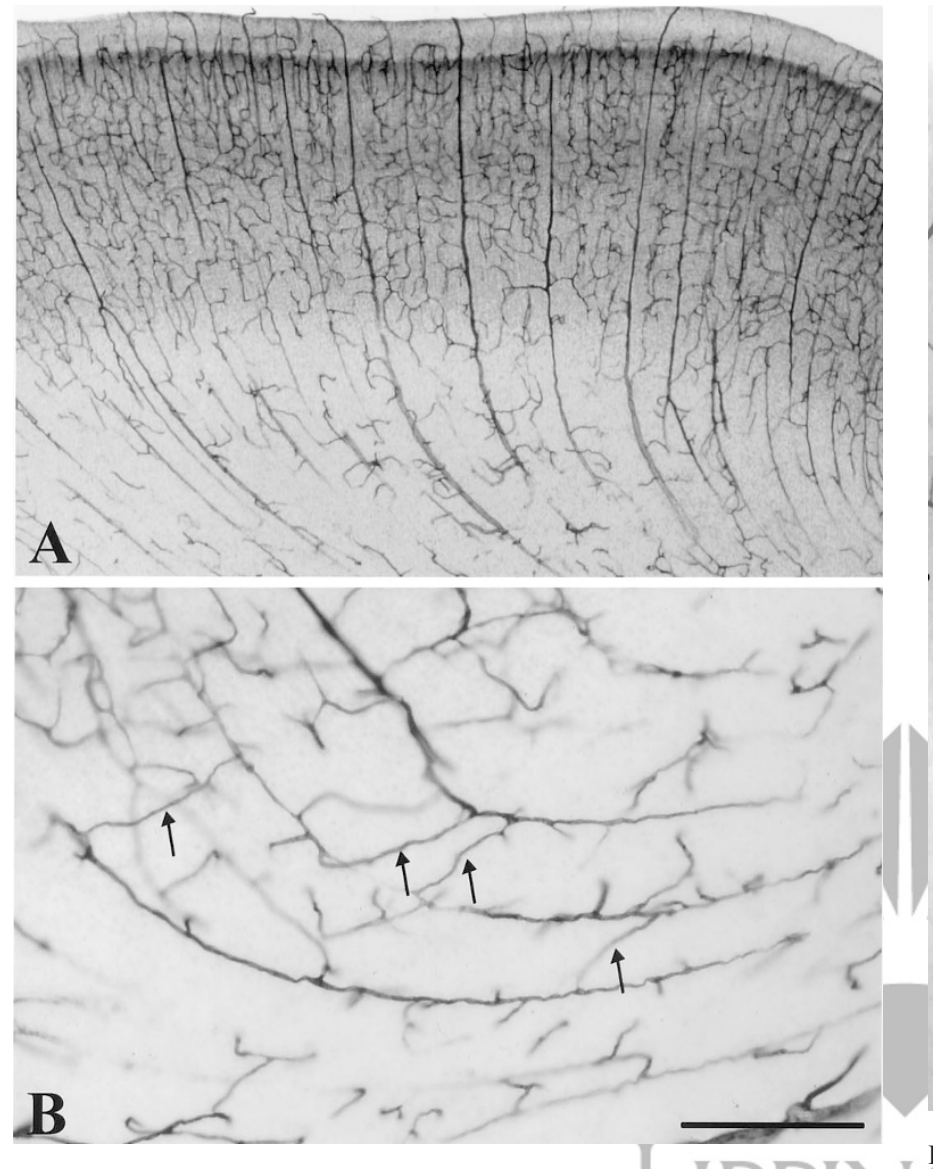

Figure 3. AP enzyme histochemistry of $100-\mu \mathrm{m}$-thick celloidin section from brain of a $28 \mathrm{wk}$ postconception neonate that survived $1 \mathrm{~d}$. (A) The sections illustrates the cortex and subcortical white matter of cerebral hemisphere. It is counterstained with hematoxylin and eosin. Parallel arterioles pass from their site of origin at the brain surface through cortex to white matter. An extensive capillary network occupies the interarteriolar space. Vascular routes from one arteriole to another are easily found. All of these routes pass through the intervening capilliary bed. $(B)$ Subcortical white matter showing three adjacent arterioles, which arise from leptomeningeal vessels, are joined by intervening capillaries (arrows). Bar $=625 \mu \mathrm{m}$ in $A ; 250 \mu \mathrm{m}$ in $B$.

measured 20-22 $\mu \mathrm{m}$ in diameter and, thus, are clearly larger than the capillaries observed in this and other microscopic fields. Capillary diameters in our preparations ranged between 6 and $11 \mu \mathrm{m}$. These shunts commonly included a transitional zone where the proximal segment narrowed abruptly into a smaller diameter channel that continued on to join the vein. In Figure $5 B$, the shunt-like connection narrows to $13 \mu \mathrm{m}$ near its juncture with the vein.

\section{DISCUSSION}

AP as a marker of cerebral afferent vessels. The utility of AP histochemistry as a marker of cerebral afferent vessels was recognized by Gomori in 1939 (19) and has been used subsequently in many studies of cerebral vascular anatomy $(12,20)$ and pathology $(10,11,21-24)$. The utility of the method was greatly enhanced by Bell and Scarrow (11), who combined the technique with celloidin embedding, which accommodated the use of sections 100-500 $\mu \mathrm{m}$ thick, thereby enhancing the three-dimensional overview of the vascular network.

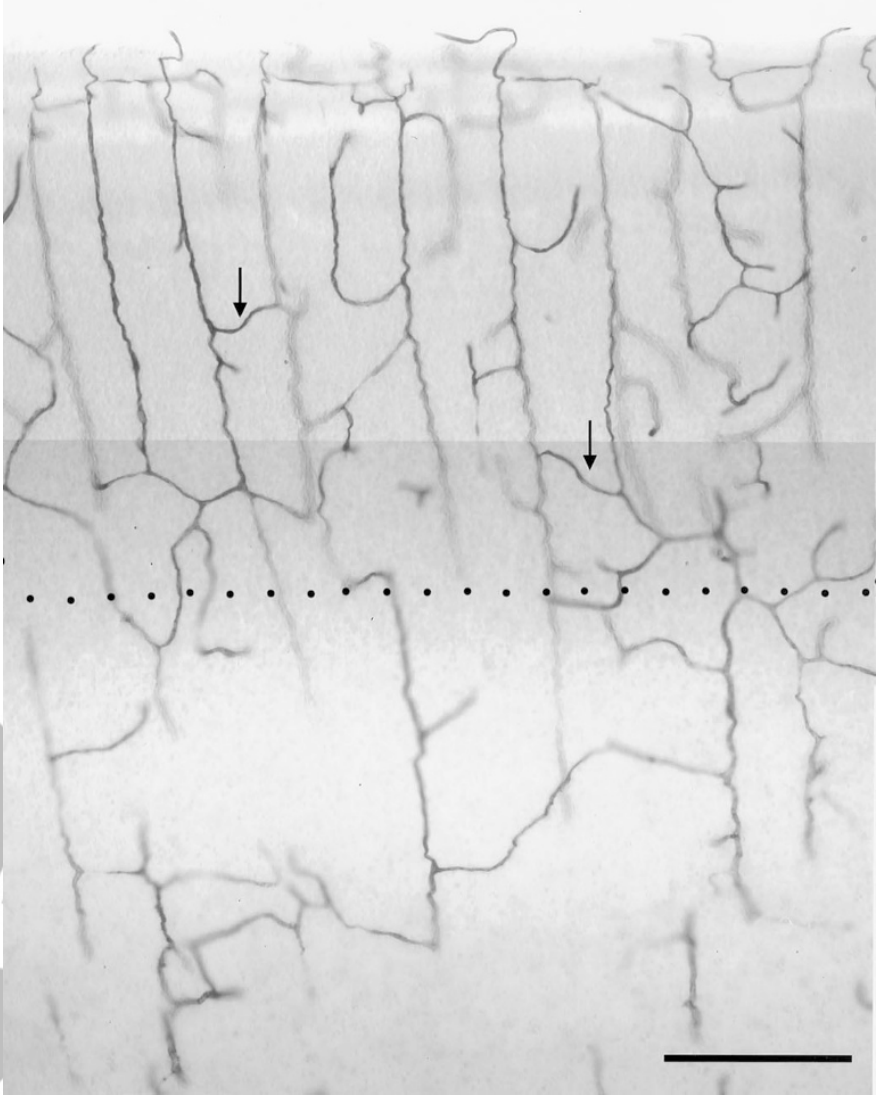

Figure 4. AP enzyme histochemistry of 100 - $\mu \mathrm{m}$-thick celloidin section from brain of a $24 \mathrm{wk}$ postconception neonate that survived $5 \mathrm{~d}$. There is a comparatively simple pattern of afferent vessels in the cortex and white matter. Short, unbranched vessels (arrows) connect adjacent arterioles. In this example, the arteriole on the left is darkly stained whereas the arteriole on the right appears lightly stained. However, the light appearance of the arteriole to the right is an illusion due to the fact that it is not in the focal plane. When the plane of focus is changed to include the arteriole on the right, it appears as darkly stained as the arteriole on the left. The dotted line represents the boundary between cortex and white matter. Bar $=250 \mu \mathrm{m}$.

The function of AP in endothelium of afferent brain vessels is not known. There are several molecules, including AP, expressed during early stages of brain vessel development in laboratory animals (25) and in humans (20). The expression has been shown to be coincident with the appearance of a functional blood-brain barrier, and it has been suggested, based upon this coincident expression pattern, that the presence of these molecules could be important for barrier function. Direct evidence to support this suggestion, however, is lacking. As discussed by Bell and Scarrow (11), AP activity should not be equated with a blood-brain barrier function inasmuch as postcapillary venules, which do not express levels of AP observed in capillaries, do have a blood-brain barrier. In addition, in many animals, skeletal muscle capillaries, which do not have a barrier, nevertheless express AP at levels comparable to the expression observed in cerebral capillaries. Furthermore, in rabbits, cerebral afferents do not express AP, even though these animals do have a blood-brain barrier [as cited in (11)]. Although the functional role of AP remains elusive, its utility as a marker of the afferent cerebral network continues to make this technique a useful research tool. 

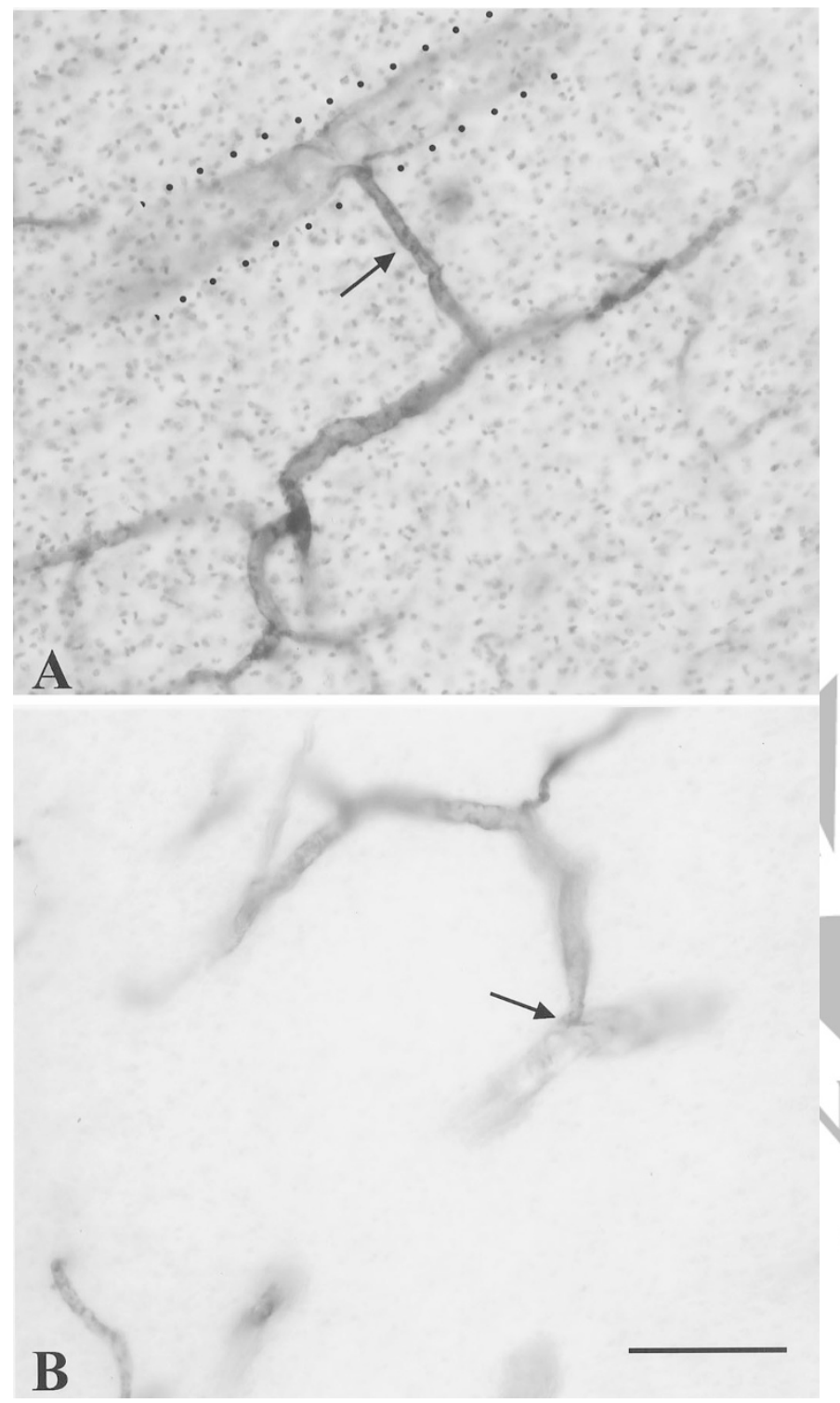

Figure 5. AP enzyme histochemistry of $100-\mu \mathrm{m}$-thick celloidin section from brain of a $24 \mathrm{wk}$ postconception neonate that survived $5 \mathrm{~d}$. (A) The section is counterstained with cresyl violet acetate/light green. In the white matter, an AP-stained vessel (arrow) larger than a capillary connects directly to an APvein. The vein in this example is made visible by the counterstain and is outlined by the two dotted lines. (B) AP-stained vessel larger than a capillary connects directly to an $\mathrm{AP}^{-}$vein. The arrow points to the juncture between the $\mathrm{AP}^{+}$vessel and the $\mathrm{AP}^{-}$vein. $\mathrm{Bar}=100 \mu \mathrm{m}$.

Arteriolar-to-arteriolar shunts in the adult brain. There has been longstanding interest in the topic of arteriolar-to-arteriolar shunts in brain parenchyma, stemming from the possibility that shunts could provide a route to reestablish an arterial blood supply to a brain region made ischemic by an embolic blockage, as occurs in stroke. Microsphere injection studies (15) indicate limited existence of arteriolar-to-arteriolar shunts. Direct evidence for shunts has come from analysis of polymer casts of cortical vascular networks (16). However, both of these experimental approaches indicate that shunts between arterioles are not a major feature of the cerebral vasculature. Furthermore, Moody et al. (12), using the AP histochemical technique in a large-scale study of adult cerebral vascular patterns, concluded that precapillary shunts between arterioles did not exist in the cortex or deeper structures of normal brain specimens. It is currently thought that capillary beds in adult human brains are continuous $(14,26)$ but that each region of the bed is supplied by a dominant arteriole that does not maintain extensive connections with neighboring arterioles other than by way of capillaries. Thus, even though arterioles are not strictly "end arterioles" in an anatomical sense, they function as such.

Arteriolar-to-venous shunts in the adult brain. There is little evidence to support the view that brain arterioles and veins are connected by shunts. The primary source of support for arteriolar-to-venous shunts is Hasegawa et al. (27), who reported finding large numbers of shunts, or "thoroughfare channels," in thick slices of both dog and human brain tissue stained to reveal arterioles, capillaries, and veins. However, Saunders and Bell (28), Duvernoy et al. (17), and Moody et al. (12) suggest that these shunts were misdiagnosed and that the structures reported as shunts were actually crossing vessels rather than joining vessels. This view is consistent with physiologic measures of arteriovenous shunting. Marcus et al. (29) determined that, after injection of 15-50 $\mu \mathrm{m}$ diameter spheres into the left atrium of dog hearts, only $1-2 \%$ of the spheres were found subsequently in the blood of the dural venous sinus. These authors concluded that large cerebral shunts are relatively few (29). Currently, it is generally accepted that brain arterioles are connected to veins only through capillary beds.

Vascular shunts in fetal brains. The vascular substrate of the fetal brain is known primarily from injection studies (30, 31 ) and from pathology literature (5). Ordinary neuropathological preparations are of limited value in following the course and branching pattern of blood vessels, because thick sections, which are necessary to demonstrate long, complex anatomical units, cannot be obtained from paraffin-embedded tissue, the medium normally used in pathology laboratories. Injection preparations that overcome the limitations of paraffin embedding are flawed because incomplete filling, air bubbles, rupture artifacts, and, most importantly for our purposes, the difficulty in discriminating between arteries and veins, complicate interpretation. Well-injected, thick sections produce a maze of vessels extraordinarily difficult to analyze because of the vessel density. In addition, injected specimens are often examined by microradiography (5). When the detector reduces the image into a two-dimensional display, i.e. $\mathrm{x}$-ray film, discriminating between branch points and crossing vessels becomes a formidable task. This problem is obviated in our thick celloidin sections because, with light microscopy, we can focus up and down through the tissue and determine branching points without ambiguity. AP histochemical staining, combined with celloidin embedding, is the most sophisticated method of brain vascular mapping. It is uniquely capable of revealing the brain vascular substrate.

Although both precapillary arteriolar-to-venous $(5,27)$ and arteriolar-to-arteriolar shunts $(27,31)$ have been reported in brains of premature babies, our investigation failed to identify these anatomical structures in all specimens except one. We conclude that fetal brains exhibit a circulatory pattern that 
resembles the adult pattern, where blood travels in an orderly progression from arterioles to capillaries to veins and that shunts do not typically connect parenchymal arterioles to either neighboring arterioles or veins. Shunts from arterioles to veins are not a general feature of the fetal brain from 23 wk onward. Therefore, arteriole-to-vein shunts are not likely to be a significant factor in transferring arterial blood pressure directly into the venous system. The elevated venous pressure that leads to venous rupture and germinal matrix hemorrhage more likely results from impaired venous flow through the heart and lungs caused by conditions associated with prematurity, such as respiratory distress syndrome and pneumothorax, exacerbated, possibly, by therapeutic maneuvers that increase thoracic pressure acutely $(13,26)$.

We do have what appears to be unmistakable evidence of arteriolar-to-venous shunts in one brain out of the 35 that were examined. This case exhibits multiple examples of $\mathrm{AP}^{+}$vessels larger than capillaries connecting to $\mathrm{AP}^{-}$vessels, which, in addition to being $\mathrm{AP}^{-}$, exhibit the "sac-like" morphology typical of veins. This particular baby, born at $23 \mathrm{wk}$ gestation, was afflicted with bilateral pneumothoraces and this condition may have contributed to the formation of these unusual vascular profiles. They may reflect a pathologic condition. For example, pneumothoraces would elevate thoracic pressure and thereby impede venous return from the cranium. Elevated intracranial venous pressure could cause swelling of all cerebral parenchymal vessels, including those that connect arterioles to veins. It may be the pathologically swollen connecting vessels that appear as precapillary shunts in our preparations. The examples of shunts in our one preparation are so prominent and so easily detected in our specimens that it is improbable that we have simply overlooked similar structures in the remainder of our specimens.

The increasing anatomical complexity of vascular units. Our specimens highlight the increasing anatomical complexity of the brain vascular system that ensues as gestation proceeds from $23 \mathrm{wk}$, about the earliest stage compatible with survival, to $>30 \mathrm{wk}$, where the vascular architecture resembles that of term babies. By 33 wk gestation, complex capillary networks are in place and blood must flow through these capillaries before passing on to veins. The picture is considerably different in the youngest specimens. At $23 \mathrm{wk}$ gestation, extensive capillary networks are not yet present. Neighboring vessels, either arterioles or veins, extending from pial vessels toward the lateral ventricle, are often seen to be joined by straight, unbranched channels. These simple connecting channels are the diameter of capillaries and, therefore, would not be considered shunts. However, we speculate that blood passing through them would not exhibit a drop in pressure equivalent to the drop observed when blood in older specimens flows from an arteriole into an extensive capillary bed. Compared with the older specimens, in the younger specimens it appears that the venous side of the circulation is more closely and directly connected to the arterial side, and it is possible that severe fluctuations in arterial pressure that can occur during birth and in the early postnatal period, especially in the sickest babies, may be transferred to the venous circulation with less moderation of pressure levels. The anatomical simplicity of the cerebral circulatory system at 23 wk may in and of itself be a risk factor to these premature babies.

Acknowledgments. The authors thank Patricia Wood and Carolyn Cox for their expert technical assistance in the preparation of histologic specimens.

\section{REFERENCES}

1. Volpe JJ 2001 Neurology of the Newborn. WB Saunders, Philadelphia, pp 428-493

2. Volpe JJ 1997 Brain injury in the premature infant - from pathogenesis to prevention. Brain Dev 19:519-534

3. Towbin A 1968 Cerebral intraventricular hemorrhage and subependymal matrix infarction in the fetus and premature newborn. Am J Pathol 52:121-140

4. Leech RW, Kohnen P 1974 Subependymal and intraventricular hemorrhages in the newborn. Am J Pathol 77:465-475

5. Pape KE, Wigglesworth JS 1979 Haemorrhage, ischaemia and the perinatal brain. Clin Dev Med 69/70:1-185

6. Pasternak JF, Groothuis DR, Fischer JM, Fischer DP 1983 Regional cerebral blood flow in the beagle puppy model of neonatal intraventricular hemorrhage: studies during systemic hypertension. Neurology 33:559-566

7. Perlman JM, Goodman S, Kreusser KL, Volpe JJ 1985 Reduction in intraventricular hemorrhage by elimination of fluctuating cerebral blood-flow velocity in preterm infants with respiratory distress syndrome. N Engl J Med 312:1353-1357

8. Volpe JJ 1989 Intraventricular hemorrhage in the premature infant-current concepts. Part I. Ann Neurol 25:3-11

9. Nakamura Y, Okudera T, Fukuda S, Hashimoto T 1990 Germinal matrix hemorrhage of venous origin in preterm neonates. Hum Pathol 21:1059-1062

10. Ghazi-Birry HS, Brown WR, Moody DM, Challa VR, Block SM, Reboussin DM 1997 Human germinal matrix: venous origin of hemorrhage and vascular characteristics. AJNR Am J Neuroradiol 18:219-229

11. Bell MA, Scarrow WG 1984 Staining for microvascular alkaline phosphatase in thick celloidin sections of nervous tissue: morphometric and pathological applications. Microvasc Res 27:189-203

12. Moody DM, Bell MA, Challa VR 1990 Features of the cerebral vascular pattern that predict vulnerability to perfusion or oxygenation deficiency: an anatomic study. AJNR Am J Neuroradiol 11:431-439

13. Mayer PL, Kier EL 1991 The controversy of the periventricular white matter circulation: a review of the anatomic literature. AJNR Am J Neuroradiol 12:223-228

14. Nelson MDJ, Gonzalez-Gomez I, Gilles FH 1991 Dyke Award. The search for human telencephalic ventriculofugal arteries. AJNR Am J Neuroradiol 12:215-222

15. Kennady JC, Taplin GV 1967 Shunting in cerebral microcirculation. Am Surg 33:763-771

16. Anderson BG, Anderson WD 1978 Shunting in intracranial microvasculature demonstrated by SEM of corrosion-casts. Am J Anat 153:617-624

17. Duvernoy HM, Delon S, Vannson JL 1981 Cortical blood vessels of the human brain. Brain Res Bull 7:519-579

18. Moody DM, Brown WR, Challa VR, Block SM 1994 Alkaline phosphatase histochemical staining in the study of germinal matrix hemorrhage and brain vascular morphology in a very-low-birth-weight neonate. Pediatr Res 35:424-430

19. Gomori G 1939 Microtechnical demonstration of phosphatase in tissue sections. Proc Soc Exp Biol Med 42:23-26

20. Anstrom JA, Brown WR, Moody DM, Thore CR, Challa VR, Block SM 2002 Temporal expression pattern of cerebrovascular endothelial cell alkaline phosphatase during human gestation. J Neuropathol Exp Neurol 61:76-84

21. Bell MA, Ball MJ 1981 Morphometric comparison of hippocampal microvasculature in ageing and demented people: diameters and densities. Acta Neuropathol 53:299318

22. Moody DM, Brown WR, Challa VR, Stump DA, Reboussin DM, Legault C 1995 Brain microemboli associated with cardiopulmonary bypass: a histologic and magnetic resonance imaging study. Ann Thorac Surg 59:1304-1307

23. Moody DM, Brown WR, Challa VR, Ghazi-Birry HS, Reboussin DM 1997 Cerebral microvascular alterations in aging, leukoaraiosis, and Alzheimer's disease. Ann N Y Acad Sci 826:103-116

24. Scharrer E 1950 A technique for the demonstration of the blood vessels in the developing central nervous system. Anat Rec 107:319-327

25. Risau W, Hallmann R, Albrecht U 1986 Differentiation-dependent expression of proteins in brain endothelium during development of the blood-brain barrier. Dev Biol 117:537-545

26. Kuban KC, Gilles FH 1985 Human telencephalic angiogenesis. Ann Neurol 17:539548

27. Hasegawa T, Ravens JR, Toole JF 1967 Precapillary arteriovenous anastomoses. "Thoroughfare channels" in the brain. Arch Neurol 16:217-224

28. Saunders RL, Bell MA 1971 X-ray microscopy and histochemistry of the human cerebral blood vessels. J Neurosurg 35:128-140

29. Marcus ML, Heistad DD, Ehrhardt JC, Abboud FM 1976 Total and regional cerebral blood flow measurement with 7-10-, 15-, 25-, and 50-mum microspheres. J Applied Physiol 40:501-507

30. Takashima S, Mito T, Ando Y 1986 Pathogenesis of periventricular white matter hemorrhages in preterm infants. Brain Dev 8:25-30

31. Nakamura Y, Okudera T, Hashimoto T 1991 Microvasculature in germinal matrix layer: its relationship to germinal matrix hemorrhage. Mod Pathol 4:475-480 